\title{
Cost analysis of standard Sanger sequencing versus next generation sequencing in the ICONIC study
}

Patel Nishma ${ }^{1}$, Ferns R. Bridget ${ }^{2}$, Nastouli Eleni ${ }^{2}$, Kozlakidis Zisis ${ }^{3}$, Kellam Paul ${ }^{4}$, Morris Stephen ${ }^{1}$

1. Department of Applied Health Research (P Nishma MSc, M Stephen PhD), and 3 Division of Infection and Immunity (K Zisis PhD), University College London, London, UK; 4 Division of Infectious Diseases, Imperial College London, UK (K Paul PhD); and 2 Clinical Microbiology and Virology, University College London Hospitals NHS Foundation Trust, London, UK (F R Bridget PhD, N Eleni MD)

\section{Introduction}

HIV and hepatitis $\mathrm{C}$ virus (HCV) are a major cause of morbidity and mortality, and both viruses contain high genomic variation. To date, viral gene sequencing has been handled by standard Sanger sequencing (SSS) for the detection of specific drug-resistance determinants for HIV and HCV. However, SSS-derived information is limited. By contrast, full-length viral gene sequences when linked to clinical data might influence the monitoring of drug resistance to optimally guide treatment, identify sources of viral transmissions within health-care settings, and track emerging epidemics. The ICONIC (Infection Response through Virus Genomics) study aims to introduce a novel method called next generation sequencing (NGS) within UK health-care settings, testing potential implementation in routine practice. With use of samples within established diagnostic laboratory workflows, NGS has the potential to produce higher informational content than SSS and report in a timely fashion. However, economic evidence for this emerging method is scarce.

\section{Aims}

To use the examples of HIV and HCV to compare the cost of NGS versus SSS.

\section{Methods}

We performed a bottom-up cost analysis using published, genomic-testing, costing templates to estimate the mean cost per sample for SSS and NGS methods over a 1 year period at a major London hospital. Data on resource use associated with genomic testing were based on estimates from individual sample data, routinely collected from a UK population.

\section{Results}

With SSS, mean cost per sample, including operating costs, was $£ 178$ for HCV (2080 samples) and $£ 79$ for HIV (520). Mean cost per sample with NGS was $£ 119$ (2207 samples, including operating costs), generating a cost saving of $£ 59$ for HCV and a surplus of $£ 40$ for HIV.

\section{Conclusions}

Although this method is still research based and prices vary widely, our results demonstrated a broad NGS and SSS cost equivalence.

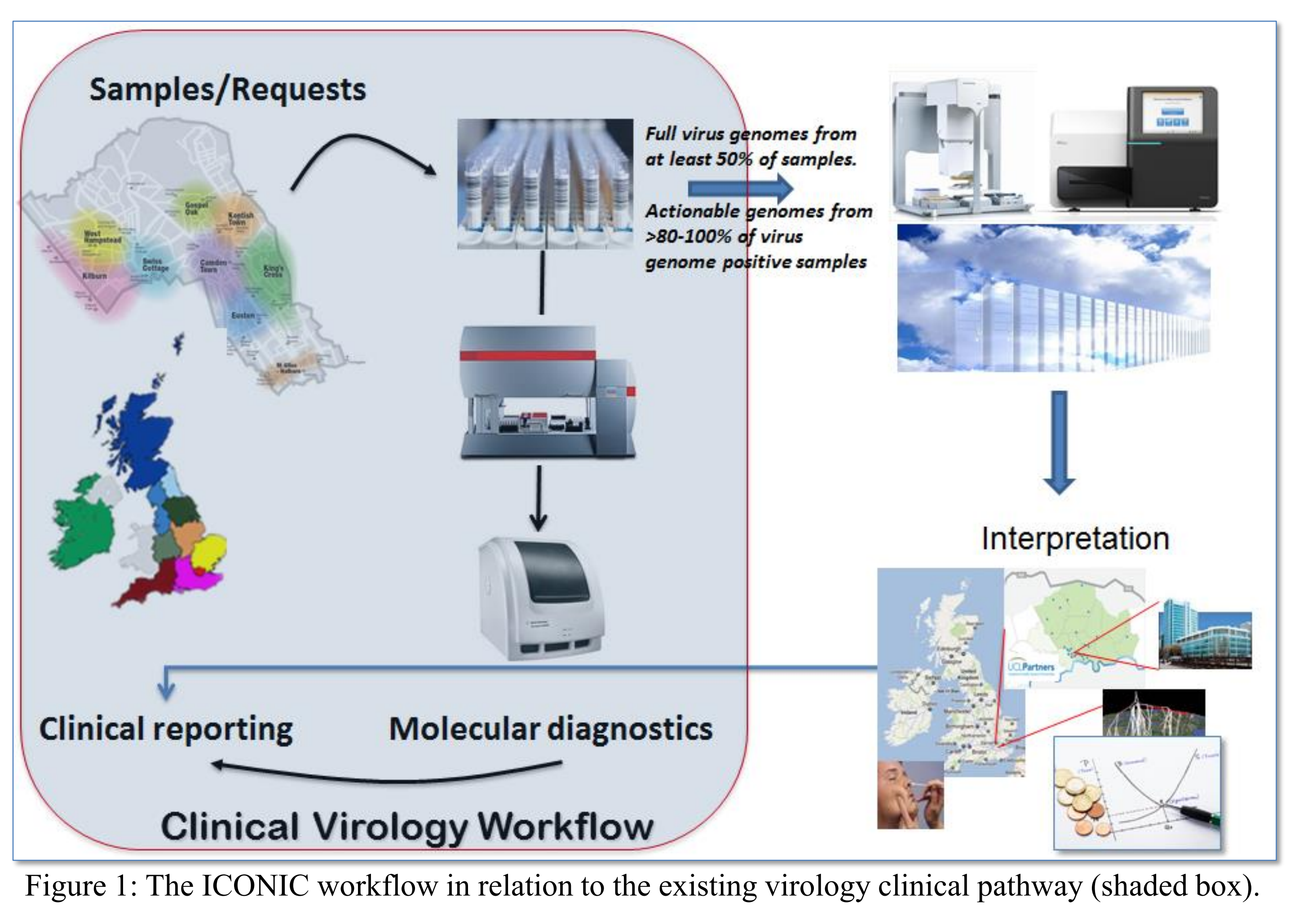

\begin{abstract}
Viral infections represent a significant burden to the NHS and a continuing threat to human health with national treatment and care costs for influenza pandemics for example in excess of £2billion. The effect of virus outbreaks is difficult to calculate because of the often very constraint time windows in which patients and their linked data need to be processed, interpreted and actioned upon and the high number of related patient co-morbidities.

Until recently the investigation of viral transmission during an outbreak was challenging as molecular typing models such as Sanger Sequencing (SSS) had low discriminatory power and their informative value on infection control measures was limited, resulting in reduced outbreak containment and increasing healthcare costs. This is set to change with Next Generation Sequencing (NGS) which provides the maximum possible discrimination between closely related viral strains. Here we present the preliminary results from the Infection Control through Viral Genomics (ICONIC) project, a flagship Health Innovation Challenge Fund project with the aim or introducing NGS processes into routine NHS operations for the effective detection and surveillance of viruses and improved stratification of patients.
\end{abstract}

Table 1: HCV and HIV bottom up cost per sample

\begin{tabular}{|c|c|c|c|c|c|c|c|c|c|c|}
\hline \multirow{3}{*}{ Sample size } & \multicolumn{5}{|c|}{ HCV cost category } & \multicolumn{5}{|c|}{ HIV cost category } \\
\hline & \multicolumn{5}{|c|}{2080} & \multicolumn{5}{|c|}{520} \\
\hline & Equipment & Consumables & Staff & Miscellaneous & All categories & Equipment & Consumables & Staff & Miscellaneous & All categories \\
\hline \multicolumn{11}{|l|}{ Stage } \\
\hline Clinical sample booking in (plasma, tissue, stool etc) & $£ 4.51$ & $£ 0.02$ & $£ 0.04$ & $£ 5.00$ & $£ 9.57$ & $£ 1.13$ & $£ 0.02$ & $£ 0.02$ & $£ 1.25$ & $£ 2.42$ \\
\hline $\begin{array}{l}\text { Clinical sample testing (Nucleic acid extraction both } \\
\text { DNA and RNA) }\end{array}$ & $£ 50.81$ & $£ 3.08$ & $£ 2.58$ & - & $£ 56.47$ & $£ 12.70$ & $£ 3.08$ & $£ 0.64$ & - & $£ 16.42$ \\
\hline $\begin{array}{l}\text { Reverse transcription and genome amplification by } \\
\text { targeted PCR to generate amplicon 3-6 hours }\end{array}$ & $£ 4.18$ & $£ 12.76$ & $£ 2.58$ & $£ 0.64$ & $£ 20.16$ & $£ 1.05$ & $£ 16.77$ & $£ 0.84$ & - & $£ 18.66$ \\
\hline Standard Sanger Sequencing run time 5-8 hours & $£ 37.90$ & $£ 12.16$ & $£ 1.16$ & - & $£ 51.22$ & $£ 9.47$ & $£ 12.83$ & $£ 0.58$ & - & $£ 22.88$ \\
\hline Standard sanger sequencing data analysis 2 hours & $£ 0.64$ & - & $£ 1.55$ & - & $£ 2.19$ & $£ 0.16$ & - & $£ 1.55$ & $£ 0.16$ & $£ 1.87$ \\
\hline Total (before overheads) & $£ 98.84$ & $£ 28.01$ & $£ 7.91$ & $£ 5.64$ & $£ 140.40$ & $£ 24.51$ & $£ 32.69$ & $£ 3.63$ & $£ 1.41$ & $£ 62.24$ \\
\hline$\%$ total cost & $70 \%$ & $20 \%$ & $6 \%$ & $4 \%$ & - & $39 \%$ & $£ 52 \%$ & $6 \%$ & $2 \%$ & - \\
\hline Total (including overheads at $27.5 \%$ ) & $£ 125.01$ & $£ 10.08$ & $£ 35.72$ & $£ 7.20$ & $£ 178.00$ & $£ 31.25$ & $£ 41.68$ & $£ 4.62$ & $£ 1.80$ & $£ 79.35$ \\
\hline
\end{tabular}

